\title{
Spectroscopy study of the dynamics of the transencephalic electrical impedance in the perinatal brain during hypoxia
}

\author{
Fernando Seoane ${ }^{1,2}$, Kaj Lindecrantz $z^{1}$, Torsten Olsson ${ }^{2}$, \\ Ingemar Kjellmer $^{3}$, Anders Flisberg ${ }^{3}$ and Ralph Bågenholm ${ }^{3}$ \\ ${ }^{1}$ School of Engineering, University College of Borås, Borås, Sweden \\ ${ }^{2}$ Department of Signal and Systems, Chalmers University of Technology, Gothenburg, Sweden \\ ${ }^{3}$ Department of Pediatrics, Göteborg University, The Queen Silvia Children's Hospital, \\ Gothenburg, Sweden
}

Received 10 April 2005, accepted for publication 13 July 2005

Published 8 August 2005

Online at stacks.iop.org/PM/26/849

\begin{abstract}
Hypoxia/ischaemia is the most common cause of brain damage in neonates. Thousands of newborn children suffer from perinatal asphyxia every year. The cells go through a response mechanism during hypoxia/ischaemia, to maintain the cellular viability and, as a response to the hypoxic/ischaemic insult, the composition and the structure of the cellular environment are altered. The alterations in the ionic concentration of the intra- and extracellular and the consequent cytotoxic oedema, cell swelling, modify the electrical properties of the constituted tissue. The changes produced can be easily measured using electrical impedance instrumentation. In this paper, we report the results from an impedance spectroscopy study on the effects of the hypoxia on the perinatal brain. The transencephalic impedance, both resistance and reactance, was measured in newborn piglets using the four-electrode method in the frequency range from $20 \mathrm{kHz}$ to $750 \mathrm{kHz}$ and the experimental results were compared with numerical results from a simulation of a suspension of cells during cell swelling. The experimental results make clear the frequency dependence of the bioelectrical impedance, confirm that the variation of resistance is more sensitive at low than at high frequencies and show that the reactance changes substantially during hypoxia. The resemblance between the experimental and numerical results proves the validity of modelling tissue as a suspension of cells and confirms the importance of the cellular oedema process in the alterations of the electrical properties of biological tissue. The study of the effects of hypoxia/ischaemia in the bioelectrical properties of tissue may lead to the development of useful clinical tools based on the application of bioelectrical impedance technology.
\end{abstract}


Keywords: bioimpedance, brain damage, cellular oedema, cerebral monitoring, perinatal asphyxia

(Some figures in this article are in colour only in the electronic version)

\section{Introduction}

Hypoxia/ischaemia is responsible for many disorders, disabilities and deaths related to the nervous system as a result of severe lack of oxygen and/or cerebral circulation failure. For instance, up to $48 \%$ of all patients suffer from cognitive dysfunction after cardiac surgery with imposed cardiac arrest (Toner et al 1998) and up to $1.5 \%$ of newborns suffer from perinatal asphyxia (Legido et al 2000). This high incidence rate of hypoxic/ischaemic brain damage not only influences dramatically the life of thousands of affected people and their families, but also entails a huge economic burden for society, billions of euros every year worldwide.

Perinatal asphyxia is one of the diseases related to hypoxic/ischaemic brain damage and it is the most significant cause of mortality, neuro-developmental disability and long-term neurological morbidity in newborn infants. Neural rescue therapies have been tested on animals with satisfactory results (Gunn et al 1998) and clinical trials of hypothermia therapy are in progress on humans. Even when a successful therapy may allow the recovery of the affected patients as a requirement prior to therapy initiation, the hypoxic situation needs to be clearly and efficiently detected. Moreover, the time window between insult and start of therapy appears to be narrow (Vannucci and Perlman 1997, Roelfsema et al 2004).

\subsection{Hypoxia and cellular oedema}

Hypoxia/ischaemia is an important and common cause of cell injury. It affects the cellular metabolism, impinging on the aerobic oxidative respiration of the cell (Cotran et al 1989). Lack of oxygen in the cell forces the cell to resort to anaerobic metabolism with glycogenglucose breakdown halted at the lactate level. The reduced capability of the cell to produce energy forces the cell membrane to lose some of its regulation and active transport functions. This leads to a rapid accumulation of osmotic active products in the intracellular space, an accumulation accompanied by an isosmotic increment in water and resulting in an inevitable intracellular swelling. This intracellular swelling is denominated cellular or cytotoxic oedema (Klatzo 1994) and it characterizes the early phase of hypoxia/ischaemia.

\subsection{Electrical bioimpedance}

Biological tissue is a dielectric conductive material and electrical current passes through the tissue when a difference of electric potential is applied to the tissue in a closed electric circuit. Biological tissue is often considered as an aggregation of cells, with a semi-permeable membrane containing the intracellular fluid, surrounded by the extracellular fluid. Both intracellular and extracellular fluids are conductive, therefore exhibiting a certain resistance, and the cellular membrane acts as a capacitor, figure 1 .

\subsection{Effects of cell swelling on the bioelectrical impedance of the tissue}

Cell swelling causes cellular oedema, which implies a redistribution of fluids between the intracellular and extracellular spaces. This fluid redistribution causes changes in the structure 


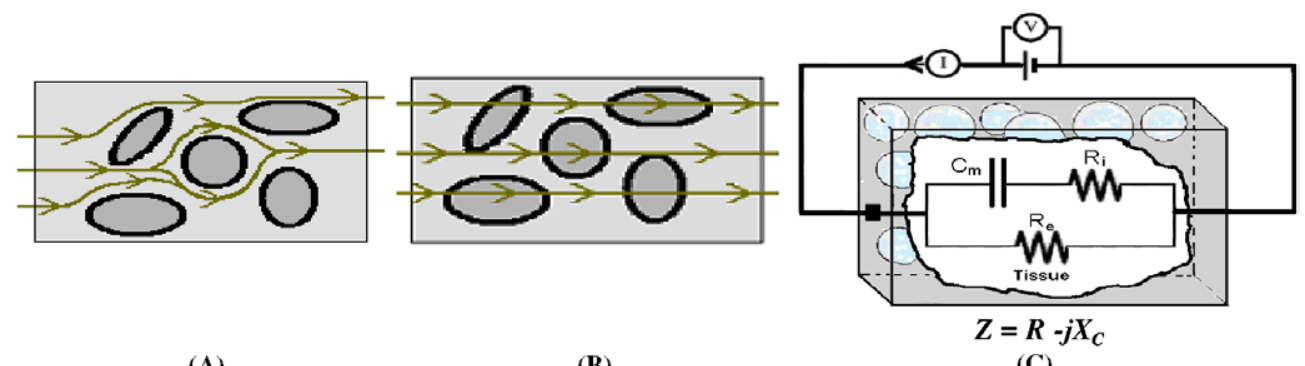

(A)

(B)

(C)

Figure 1. (A) and (B) show the pathways of the electrical current through biological tissue; (A) low frequency, (B) high frequency. (C) shows the electrical circuit model equivalent of biological tissue; three elements $2 \mathrm{R}-1 \mathrm{C}$ parallel type.

of the tissue, resulting in changes in the electrical bioimpedance (Van Harreveld 1957). The electrical impedance consists of two components: resistance and reactance, and both depend on the shape of the cells, tissue structure and tissue composition. Therefore, our working hypothesis is that during cell swelling both of them may be modified notably and in theory substantial changes are expected, especially in the reactive part.

Previous experimental studies, focused on hypoxic/ischemic brain damage, have successfully confirmed the association between hypoxic/ischemic events and changes of the electrical bioimpedance in the brain (Van Harreveld 1957, Williams et al 1991, Holder 1992, Lingwood et al 2002).

\subsection{Multi-frequency measurements in brain hypoxa/ischaemia}

Multi-frequency analysis of brain electrical impedance during hypoxia/ischaemia has been used only once before, to our knowledge, in Lingwood et al (2002). In such study, multifrequency measurements of resistance and reactance were performed and using the Cole analysis the resistance at zero frequency $\left(R_{0}\right)$ was estimated. The dynamics of the estimated $R_{0}$ value were reported but no multi-frequency impedance data were included in the results.

In agreement with our working hypothesis, in this study we take the step of studying the dynamics of the impedance during normoxia followed by an induced hypoxic event and further during a phase of reoxygenation. We study the measured complex impedance, both resistance and reactance, and the observed frequency dependence on the measurements' frequency range.

In order to verify that the measured variations in impedance are in fact related to hypoxic cell swelling, the variations are interpreted in relation to the model of biological tissue as a suspension of spherical cells, figure 2 and equation (1) from Cole (1928).

$$
Z=r_{\mathrm{e}} \frac{(1-f) r_{\mathrm{e}}+(2+f)\left(r_{i}+\frac{Z_{\mathrm{m}}}{a}\right)}{(1+2 f) r_{\mathrm{e}}+2(1-f)\left(r_{i}+\frac{Z_{\mathrm{m}}}{a}\right)}
$$

where $Z$ is the specific impedance of tissue in $\Omega \mathrm{cm}, r_{\mathrm{e}}$ is the resistivity of extracellular fluid in $\Omega \mathrm{cm}, r_{i}$ is the resistivity of cytoplasm in $\Omega \mathrm{cm}, z_{\mathrm{m}}$ is the surface membrane impedivity in $\Omega \mathrm{cm}^{2}, a$ is the cell radius in $\mathrm{cm}$ and $f$ is the volume factor of concentration of cells.

\section{Methodology}

A bioimpedance spectroscopy study of biological tissue during cell swelling has been performed. An experiment with live animals was performed to invasively measure 


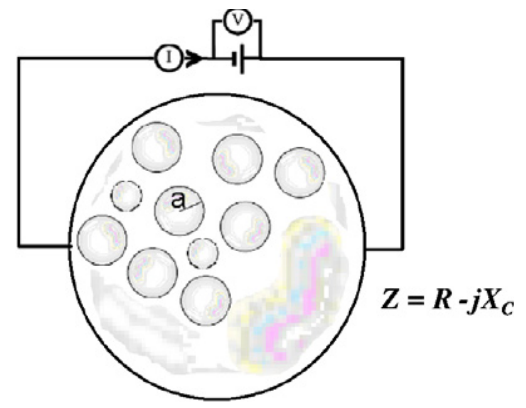

Figure 2. Suspension of spherical cells in a conductive fluid. Equation (1) shows the equivalent electrical impedivity (specific impedance) of a suspension of spherical cells.

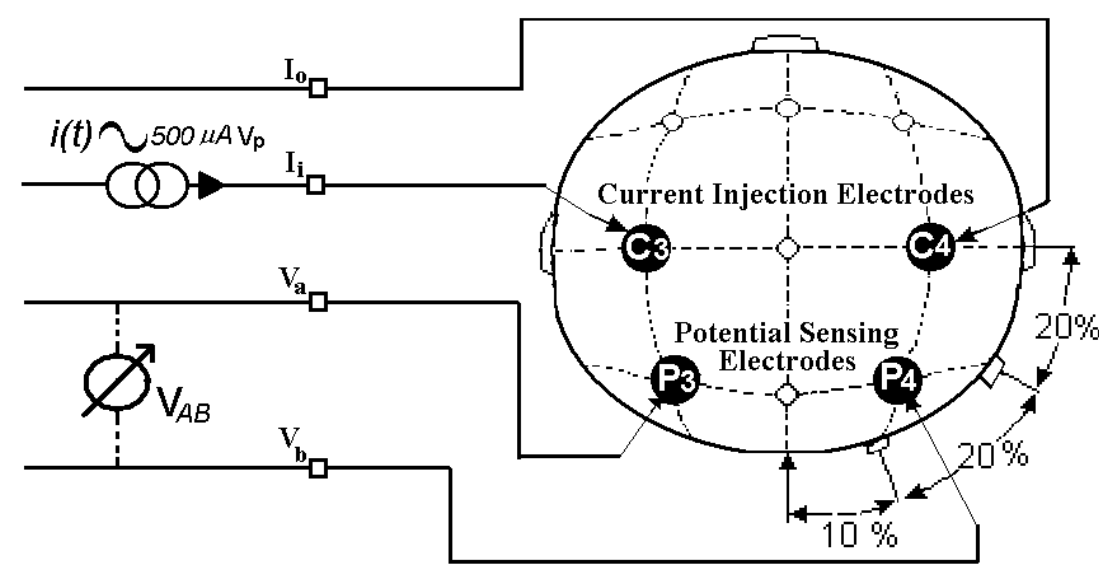

Figure 3. The four-electrode method and the placement of electrodes following the standard 10-20 system on the head of a piglet. Two electrodes inject the current while the resulting voltage through the tissue is measured with the other two. Current injecting electrodes are placed on $\mathrm{C} 3$ and $\mathrm{C} 4$ and potential-sensing electrodes are placed on P3 and P4.

transencephalic electrical impedance on piglets before, during and after hypoxia. Numerical calculations based on the model of tissue as a suspension of cells were performed to calculate the effective behaviour of the electrical bioimpedance of tissue regarding the frequency, tissue structure and radius of the cells.

The performed animal experimentation was approved by the Ethics Committee for Animal Research of Göteborg University.

\subsection{Animal preparation and induced hypoxia}

Newborn pigs, 1-4 days old, were anaesthetized with ketamine for induction and chloral hydrate for maintenance and then ventilated to maintain normal blood gases. Transencephalic impedance was recorded together with arterial blood pressure, heart rate and electroencephalic activity (EEG). After a $1 \mathrm{~h}$ long control period, $45 \mathrm{~min}$ of severe hypoxia was instituted by decreasing oxygen in the inhaled gas mixture to $6 \%$ to induce loss of EEG activity. Then oxygen was added to the gas mixture and normal oxygenation was maintained for the following $16 \mathrm{~h}$. The subjects were killed immediately at the end of the experiments by a lethal overdose of pentothal. 


\subsection{Measurement of transencephalic impedance}

Four burr holes were drilled through the scalp in positions P3, P4, C3 and C4; see figure 3. Silver rod electrodes of $2.5 \mathrm{~mm}$ diameter with rough surface were screwed into the holes with the surface resting on the dura. The electrical bioimpedance was measured using a frequency selectable custom-made impedance meter (Jakobsson 2000), based on the four-electrode current-injection/voltage-measurement method (Ackmann et al 1984). The impedance meter was calibrated with resistive loads and Cole phantoms, 2R1C parallel type, obtaining a margin error of $\pm 0.5 \Omega$ up to $650 \mathrm{kHz}$ and $+1.5 /-0.7 \Omega$ to $1300 \mathrm{kHz}$.

A symmetric sinusoidal current of $500 \mu \mathrm{A}$ peak value was applied for continuous electric stimulation. The current was injected through the current electrodes placed on C3 and C4. The complex electrical bioimpedance, resistance and reactance, was measured from potentialsensing electrodes placed on P3 and P4.

The dynamics of the resistance was studied in seven subjects at the frequencies $50 \mathrm{kHz}$ and $200 \mathrm{kHz}$. A more elaborate study was performed in two subjects where the measurements were made in the frequency range from 20 to $750 \mathrm{kHz}$. In these two subjects the impedance, resistance and reactance, was measured and the results were compared with the suspension of cells model.

\subsection{Numeric calculations on the suspension of cells model}

The impedivity of a suspension of spherical cells was calculated using the expression derived by Cole (1928) applying the articles 313 and 314 of Maxwell's treatise (Maxwell 1873). The impedivity obtained was used to calculate the impedance of a cylindrical conductor. The limits and accuracy in the calculation of the electrical bioimpedance of tissue modelled as a suspension of cells have been studied and reported by Cole (Cole et al 1969, Cole 1976).

$$
f=\frac{N_{\mathrm{c}} \times 4 \times \pi \times a^{3}}{3 \times V_{\mathrm{t}}}
$$

where, $f$ is the volume factor, $N_{\mathrm{c}}$ is the number of cells, $V_{\mathrm{t}}$ is the total volume of the suspension and $a$ is the radius of the cells.

Equation (1) was fitted to geometrically satisfy three dimensions using equation (2) to define the volume factor, $f$, with respect to the radius of the cells $a$. Both real and imaginary components were calculated with equation (1) and the resulting values were represented using complex impedance plots for different values of cell radii. The model represented a cylindrical portion of tissue of radius $20 \mathrm{~mm}$ and length $20 \mathrm{~mm}$ containing $10^{9}$ cells. The plasma membrane was considered an ideal capacitor. Thus, $z_{\mathrm{m}}$ in equation (1) is substituted by $\left(\mathrm{j} \omega c_{\mathrm{m}}\right)^{-1}$, where $\mathrm{j}$ is the imaginary operator $\sqrt{-1}, \omega$ is the angular frequency in radians $\mathrm{s}^{-1}$ and $c_{\mathrm{m}}$ is the surface membrane capacity in farads $\mathrm{cm}^{-2}$. The resistivity values used in the numerical calculation are electrical properties of myelinated nerve fibres extracted from Malmivuo and Plonsey (1995).

\section{Results}

The measurements performed showed that the electrical bioimpedance of the brain changes notably during and after hypoxia with respect to the normoxic baseline value.

\subsection{Dynamics of resistance-normoxic phase}

During normoxia, before the hypoxic insult was induced, the subjects $(n=7)$ presented a stable brain electrical resistance baseline for each subject, though at significantly different 
Table 1. Statistics of the measured resistance baseline prior to hypoxia. Measurements performed at $50 \mathrm{kHz}$ and $200 \mathrm{kHz}$ over a set of seven subjects.

\begin{tabular}{llllll}
\hline \multirow{2}{*}{ Subject } & \multicolumn{2}{c}{ Frequency $50 \mathrm{kHz}$} & & \multicolumn{2}{c}{ Frequency $200 \mathrm{kHz}$} \\
\cline { 2 - 3 } \cline { 5 - 6 } & Mean $(\Omega)$ & $\mathrm{SD}(\Omega)$ & & Mean $(\Omega)$ & $\mathrm{SD}(\Omega)$ \\
\hline 1 & 56.3 & 0.047 & & 52.1 & 0.037 \\
2 & 50.0 & 0.050 & & 45.2 & 0.051 \\
3 & 50.5 & 0.058 & & 44.1 & 0.062 \\
4 & 50.4 & 0.259 & & 47.3 & 0.221 \\
5 & 33.0 & 0.061 & & 20.7 & 0.067 \\
6 & 85.0 & 0.462 & & 75.8 & 0.495 \\
7 & 68.4 & 0.459 & & 58.8 & 0.203 \\
& \multicolumn{3}{c}{ Average at $50 \mathrm{kHz}$} & & Average at $200 \mathrm{kHz}$ \\
Mean & \multicolumn{3}{c}{$56.2 \Omega$} & & \multicolumn{2}{c}{$49.1 \Omega$} \\
Standard deviation & \multicolumn{2}{c}{$16.4 \Omega$} & & \multicolumn{2}{c}{$16.7 \Omega$} \\
\hline
\end{tabular}

Table 2. Statistics of the maximum variation in the resistance during hypoxia. Measurements performed at $50 \mathrm{kHz}$ over a set of seven subjects.

\begin{tabular}{llc}
\hline & \multicolumn{2}{c}{ Frequency $50 \mathrm{kHz}$} \\
\cline { 2 - 3 } Subject & Maximum $(\Omega)$ & Ratio $(\%)$ \\
\hline 1 & 15.4 & 27.0 \\
2 & 19.1 & 38.3 \\
3 & 38.4 & 76.1 \\
4 & 22.7 & 45.0 \\
5 & 7.5 & 22.6 \\
6 & 22.1 & 26.0 \\
7 & 81.0 & 118.4 \\
\hline
\end{tabular}

values. The average resistance measured at $50 \mathrm{kHz}$ was $56.2 \Omega$ with a $16.4 \Omega$ standard deviation (SD) and $49.1 \Omega$ with a $16.7 \Omega$ SD measured at $200 \mathrm{kHz}$. As it is possible to observe in table 1 , most of the subjects presented baseline values close to the average value, but one subject showed a very high baseline value, $85.0 \Omega$ at $50 \mathrm{kHz}$ and 75.8 at $200 \mathrm{kHz}$, and another subject showed a noticeably low baseline, $33.0 \Omega$ at $50 \mathrm{kHz}$ and 20.7 at $200 \mathrm{kHz}$.

\subsection{Dynamics of resistance-hypoxic phase}

During hypoxia, the transencephalic resistance increased remarkably over the established baseline. The same individual specificity observed for the value of the baseline was observed for the time evolution of the resistance measured during hypoxia.

Figure 4 shows the evolution of the resistance measured at $50 \mathrm{kHz}$ during hypoxia in each of the seven subjects. The resistance increases in every subject but with different slopes reaching different maximum values. The resistance began to increase right after hypoxia was initiated. The maximum observed variation was $81.0 \Omega$ corresponding to a relative increment of $118.4 \%$ over the normoxic baseline. The minimum observed variation was $7.5 \Omega$ corresponding to $22.6 \%$. See table 2 containing the maximum observed variation in the resistance for all the piglets. 


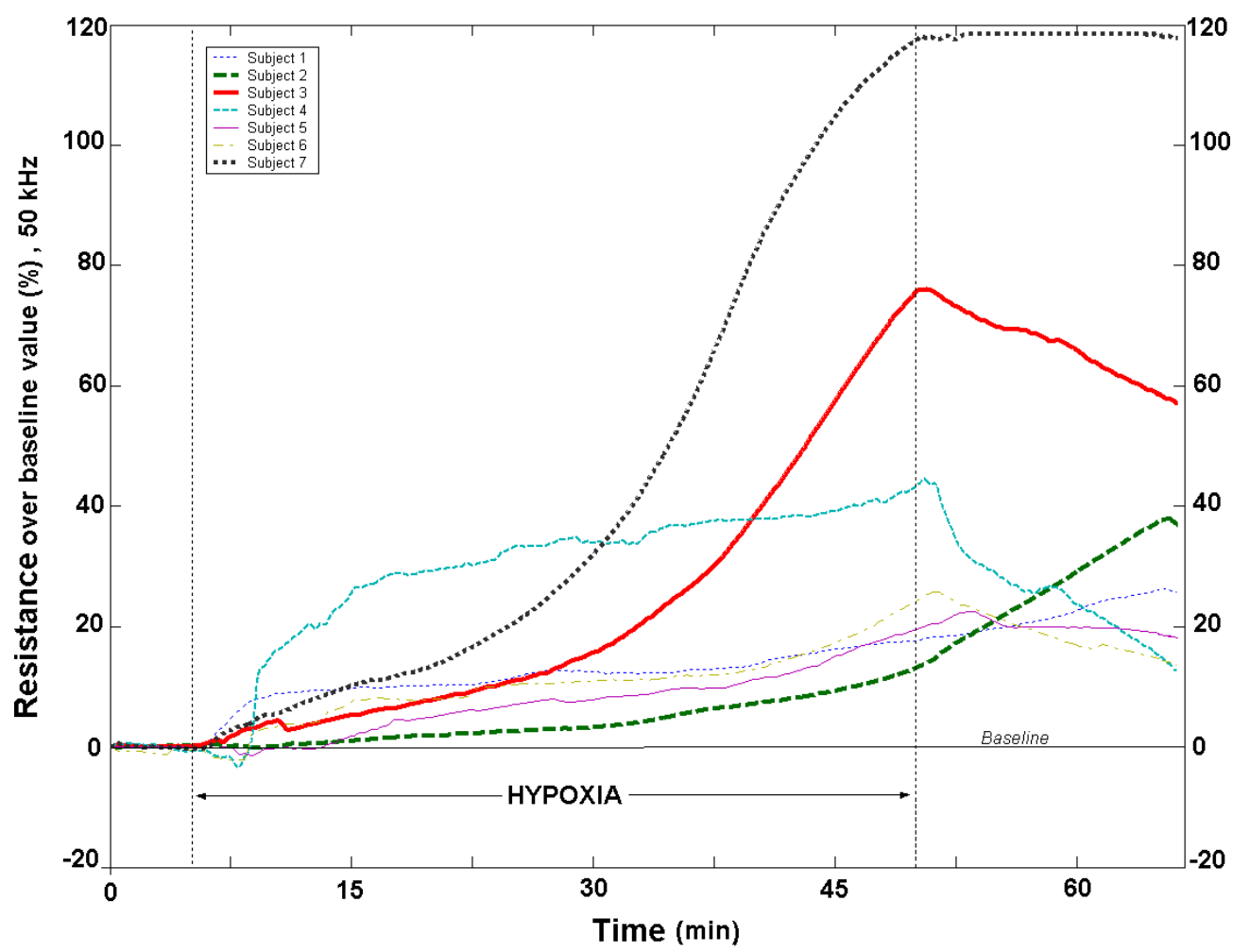

Figure 4. Time evolution of the cerebral electrical resistance in the asphyxiated piglet measured at $50 \mathrm{kHz}$ in seven different subjects. The $45 \mathrm{~min}$ hypoxic period is marked within the vertical discontinuous lines.

After the normal oxygenation was re-instituted, the resistance kept increasing for a short period of time before decreasing back to lower values. The length of such a period varies for each of the subjects; see figure 4.

The evolution of the resistance measured at $200 \mathrm{kHz}$ was identical to the evolution of the resistance at $50 \mathrm{kHz}$ for every piglet. The only difference was that the values of the magnitude and the proportional changes were slightly smaller.

\subsection{Dynamics of resistance-post-hypoxic phase}

At low frequencies, during the post-hypoxic phase three different dynamics were observed in the resistance. In the first group of piglets, the resistance decreased towards the baseline and after the baseline value was restored, the resistance value remained stable for the remaining part of the experiment. In the second group, the resistance after reaching the baseline value and keeping stable for a certain period, started increasing again. In the third group, after decreasing for a short period, the resistance started increasing again, before the baseline value was reached.

\subsection{Complex electrical bioimpedance during hypoxia}

In both piglets, the complex bioimpedance, both reactance and resistance, across the brain tissue exhibited similar changes during hypoxia and after hypoxia was instituted. Figure 5 


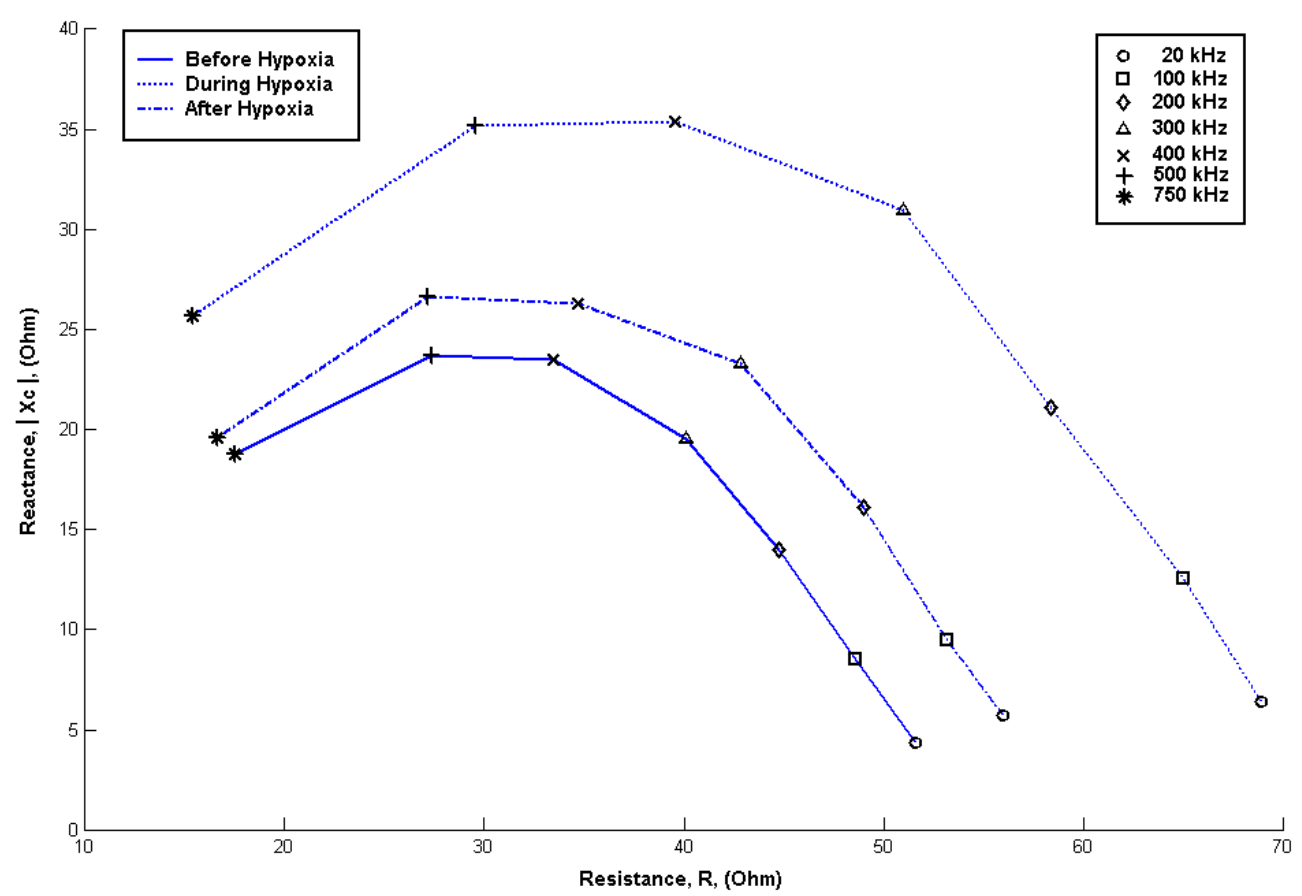

Figure 5. Plot of transencephalic electrical bioimpedance on the brain of a piglet at three different moments during hypoxic cell swelling. The complex impedance is plotted in the frequency range from $20 \mathrm{kHz}$ to $750 \mathrm{kHz}$ and from the right to the left.

shows the time evolution of the cerebral electrical impedance in one of the subjects before, during and after hypoxia. The behaviour of the cerebral impedance in the other piglets followed a similar evolution. The plots contained in figure 6 are calculated complex impedance plots, illustrating absolute reactance versus resistance over the frequency range from $20 \mathrm{kHz}$ to $750 \mathrm{kHz}$ obtained from the numerical calculations.

We observed that during hypoxia the radius of the impedance plot increased and the centre was shifted to higher values of the resistance. After oxygenation was re-instituted, the radius of the plot decreased and the centre shifted back towards the normoxic value.

The numerical results obtained from the calculations based on the suspension of cells and the experimentally measured values matched well at any frequency. Both sets of bioimpedance plots, experimental in figure 5 and numerical in figure 6 , show an analogous behaviour. The radius of the locus increases with the radius/swelling of the cells; an increase followed by a shift of the centre of the impedance locus to high values over the resistance axis.

\subsection{Effect of the frequency}

Results from the measurements showed that, during deprivation of oxygen, the reactance and the resistance changed in a different and independent manner from each other and both showed a certain frequency dependence.

The reactance increased during hypoxia at every measured frequency in the range of $20 \mathrm{kHz}-750 \mathrm{kHz}$; see figure 7(a). The maximum proportional increment in the reactance over the baseline increased with the frequency until it reached a maximum value at $300 \mathrm{kHz}$; after that it decreased with the frequency, see figure 8. 


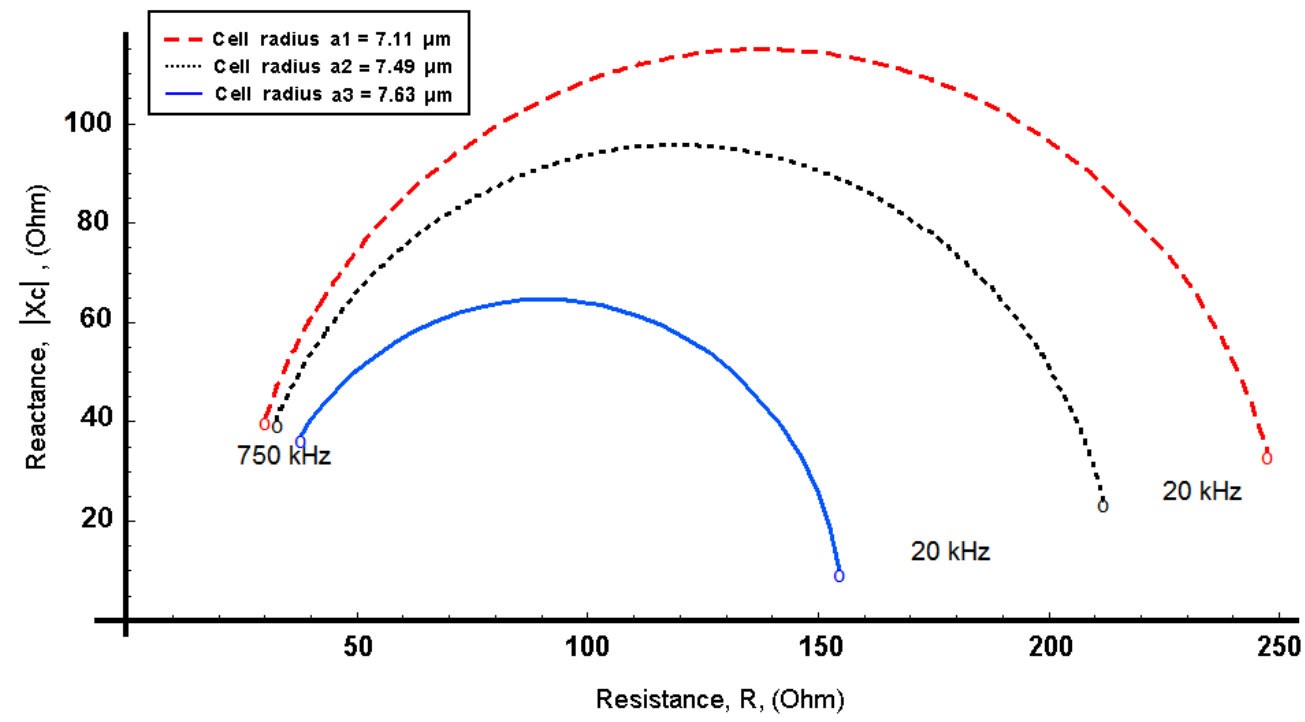

Figure 6. The complex impedance plot of a suspension of spherical cells for three different radii of cells. The suspension shape is a cylinder of radius $0.02 \mathrm{~m}$ and length $0.02 \mathrm{~m}$. According to equation (1) $r_{\mathrm{e}}=3 \Omega \mathrm{m}, r_{i}=1.1 \Omega \mathrm{m}, r_{\mathrm{m}}=\infty, C_{\mathrm{m}}=0.01 \mathrm{~F} \mathrm{~m}^{-2}$. Cells' radii $a 1=7.11 \mu \mathrm{m}$, $a 2=7.49 \mu \mathrm{m}, a 3=7.63 \mu \mathrm{m}$. The volume factor of cell concentration, $f$, is $0.60,0.70$ and 0.74 , respectively. The total number of cells in the suspension is $10^{9}$. The impedance is calculated in the frequency range from $20 \mathrm{kHz}$ to $750 \mathrm{kHz}$ and plotted from the right to the left.

The behaviour of the resistance during hypoxia showed a higher frequency dependence, increasing the most at low frequencies. The maximum proportional increment over the baseline decreased with increasing frequency and at the highest measured frequency, $750 \mathrm{kHz}$, the resistance decreased instead of increasing; see figures 7(b) and 8.

The measurements show that during hypoxia the changes in the reactance were proportionally larger than those in the resistance for the complete frequency range of the measurements, especially at high frequencies; see the comparison chart in figure 8 .

\section{Discussion}

This study confirms that cell swelling in the brain following hypoxia modifies considerably the complex electrical impedance of the brain. This is consistent with previous studies performed in different species; fetal sheep (Williams et al 1991), rats (Holder 1992) and newborn piglets (Lingwood et al 2002). These changes in the electrical bioimpedance during hypoxia occur in both resistance and reactance, real and imaginary parts of the impedance and they are approximately in concordance with numerical simulations based on tissue modelled as a suspension of cells.

\subsection{Resistance and reactance}

The reactance during the hypoxic insult changes the most and exhibits a higher sensitivity than the resistance at any measured frequency in the range of $20 \mathrm{kHz}-750 \mathrm{kHz}$. This fact is in obvious contradiction to the widely spread idea that during cell swelling alterations mainly occur in the resistance (Somjen 2001) but it is in accordance with the expected and calculated behaviour of the impedance of a suspension of spherical cells during cell swelling, increasing radius. 

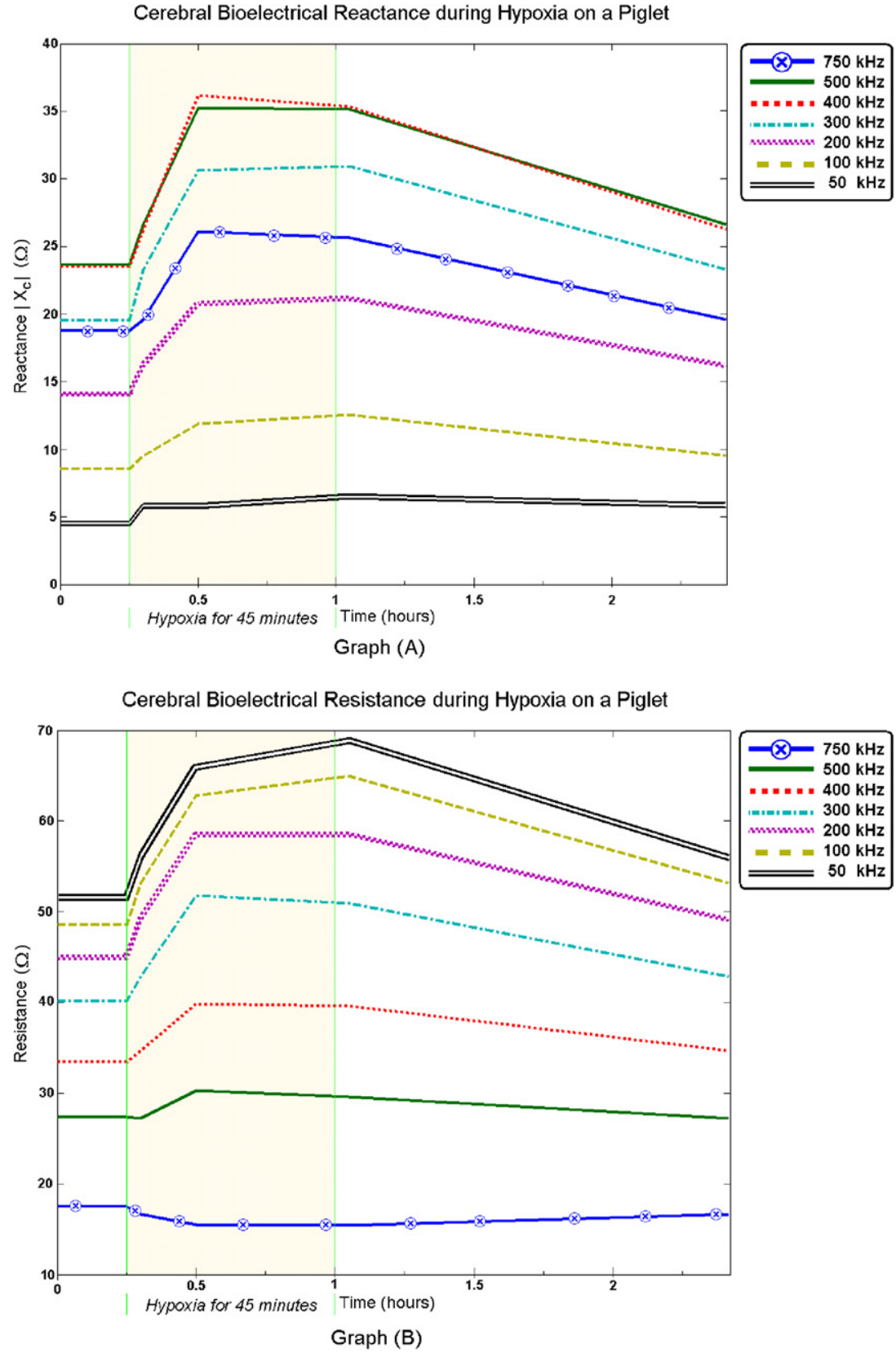

Figure 7. Evolution of transencephalic electrical impedance, reactance in (A) and resistance in (B), in a neonatal piglet during hypoxia. Measurements performed at seven frequencies in the frequency range of $20 \mathrm{kHz}-750 \mathrm{kHz}$. Hypoxia was instituted in the period between vertical markers.

\subsection{Effect of the frequency}

The reactance not only exhibits a higher sensitivity than the sensitivity observed for the resistance during hypoxia, but the sensitivity of the reactance is also much more stable than the sensitivity of the resistance over the complete frequency range. The sensitivity 
Maximum Proportional Variation of the Complex Bioimpedance during Hypoxia. A comparison chart between resistance and reactance

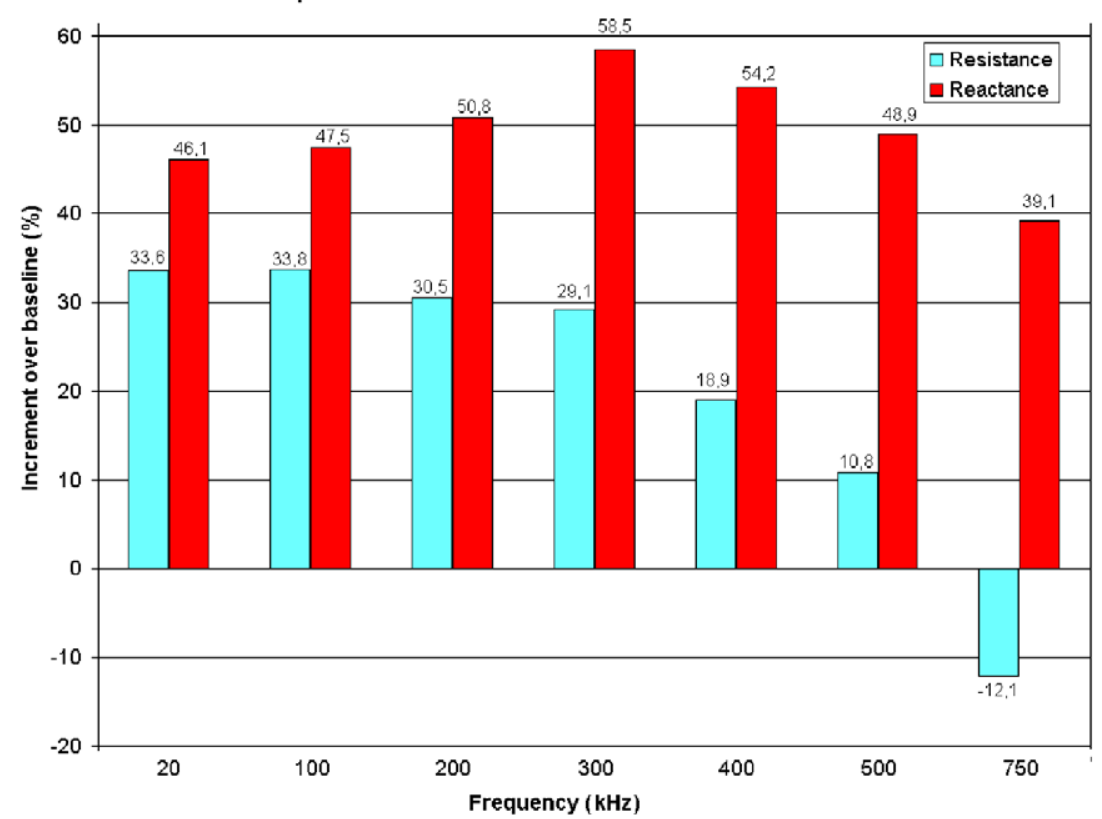

Figure 8. The comparison chart of the maximum proportional variation over the frequency of the reactance and the resistance during hypoxia in the brain tissue of the neonatal piglet.

of the reactance is also positive at any frequency and the maximum values are obtained at intermediate frequencies.

The sensitivity exhibited by the resistance changed a lot with increasing frequency. At low frequencies, the resistance changes and increases the most, as was expected from the suspension of cells model. With increasing frequency the sensitivity gradually decreases, reaching a point when the resistance decreases instead of increasing during hypoxia, resulting in a negative value of the sensitivity at high frequencies. The experimentally observed behaviour is also in accordance with the calculated behaviour of the electrical resistance of a suspension of spherical cells during cell swelling. The agreement between the theoretically expected behaviour and the experimentally obtained results is evidence to support the statement that the observed changes in the resistance at high frequencies are due to cell swelling and not as a result of measurement artefacts.

The decrease of the resistance at high frequencies during global cell swelling is probably best explained by the facts that at high frequencies the electrical current uses both intracellular and extracellular fluids to travel through the tissue and the intracellular fluid is more conductive than the extracellular fluid (Malmivuo and Plonsey 1995).

Therefore, the redistribution between extracellular and intracellular fluids in favour of the latter, occurring during cell swelling, increases the proportion of the more conductive fluid in detriment of the less conductive leaving an overall more conductive, less resistive material.

\subsection{High subject specificity}

The results obtained indicate that transencephalic electrical bioimpedance is subject specific to a very great extent. The value of the resistance, measured before hypoxia was induced, varied 
significantly between specimens. These observations are in accordance with the work reported by Lingwood et al (2003). Geometrical variations in the electrode placement between the different subjects, especially considering the small head of the piglet, may cause significant differences in the measured impedance values. Even if the placement of the four electrodes was identical between subjects, each piglet may have a slightly individual brain size that modifies in a specific way the current distribution within the conducting volume influencing the sensed measurement. Therefore, such geometric effects of the conducting volume between subjects should be considered as an important contributor to the variability of the observed baseline together with other subject specific factors, e.g., tissue morphological differences between subjects, different degrees of maturation and individual variations in the amount of cerebrospinal fluid.

The subject specificity was not only observed in the variability of the normoxic baseline value, but it was observed that, even when the cerebral impedance exhibited the same general evolution during hypoxia, the time course of the changes was highly specific to each specimen. The comparatively large variability in the individual response was also demonstrated by the different reactions of the animals during the period of re-oxygenation. Some animals recovered their normoxic impedance baseline while other animals had a secondary rise of impedance after the initial recovery. This behaviour corresponds to the previously described phases of primary and secondary energy losses (Penrice et al 1997). This should be due to the fact that the physiological system of each piglet responds in a slightly different way to the hypoxic insult and thus affects the timing of the physiological mechanism following hypoxia.

\subsection{The model of suspension of cells}

The consideration of biological tissue as a suspension of spherical cells in a conductive fluid is an approximation far from reality and to develop the numerical calculations considering the resistivity of the intracellular and extracellular space as real and frequency independent only moves the model even further away from reality. In spite of this, the model has been used for almost a century, since Fricke $(1924,1925)$, with acceptable results, and the limitations and accuracy of the different approaches have been studied and reported for many years by Cole (Cole et al 1969, Cole 1976).

It is known that the electrical conductivity of the brain tissue is anisotropic (Geddes and Baker 1967), but we have neglected that anisotropy and used an electrical isotropic model, as it has been reported by Haueisen that the anisotropy of the white and grey matter of the brain does not affect the measurements of EEG (Haueisen et al 2002).

In the simulation of the hypoxic insult on the suspension of cells model, the cell swelling effect has been considered only as an increment in the radius.

The alterations in the biochemical composition of the intra- and extracellular fluids have been neglected, considering the respective conductivities constant. Obviously, calculations based on a model considering the time alterations of local conductivities during hypoxia would be a more realistic approximation, but a study on how those conductivities change with the evolution of hypoxia has to be performed beforehand.

The morphology of the brain with the neurons and the axons forming networks in any direction is very different from a suspension of cells and it is quite unlikely that the paths that the electrical current follows through the brain tissue are similar to the paths through a suspension of spherical cells, especially in terms of homogeneities. The effect of the orientation of the biological structures on the effective impedance is clearly observed in muscular tissue, and it probably plays an important role in the impedance of brain tissue as well. Work with suspension of non-spherical cells reported by Kanai et al (2004) shows, as expected, that the 
orientation affects the total impedance of the suspension. However, the effect is significantly minimized with the increase of the volume factor of concentration of cells.

However, with the mentioned and known limitations of this simple model the reasonable agreement between the experimental data and the numerical results is remarkable and allowed us to verify that in fact the observed change of the impedance was related to hypoxic cell swelling.

\subsection{Invasive measurements}

The measurements performed in this experiment are invasive, avoiding the effect of the scalp and the skull. In non-invasive measurements, the impedance of the scalp and especially the high resistivity of the skull would reduce the effect of the alterations of internal cell swelling in the total effective transcephalic impedance. Authors (Holder 1992, Lingwood et al 2002) have reported that changes in the resistance during hypoxia are around 10-20 times smaller when measured non-invasively than when done invasively. Regarding the reactance no previous work has been found in this respect.

\subsection{About the impedance plots}

The impedance plots used in some of the figures in the results section are just regular parametric plots of reactance-resistance along the frequency; they are neither Cole plots nor Cole-Cole plots, terminology commonly misused among different authors.

A 'Cole plot' presents the evolution of the complex electrical impedance, reactance versus resistance, along the frequency as a part of a semicircular locus with the depressed centre. In the plot, the arc is segmented by the resistance axis and the impedance data are drawn along the arc from $R_{0}$ to $R_{\infty}$. The impedance data plotted are given by the equation for the impedance proposed by Cole (1940). For the application of the Cole equation a constant phase angle must be assumed for the membrane impedivity; the angle is also well indicated in the plot directly as the intersection angle between the radius from the depressed centre of the semicircle and the resistance axis (Cole 1968) or indirectly as the angle between the tangent line to the arc at the intersection point with the resistance axis (Martinsen et al 2002). A 'Cole-Cole plot' is similar to the 'Cole plot' but it contains information about complex permittivity and not about impedance (Cole and Cole 1941).

\section{Conclusion}

The consequent cell swelling following hypoxia causes measurable alterations in the electric impedance of the tissue. These alterations affect both the real and the imaginary parts, resistance and reactance, and can be measured with the four-electrode method.

Bioelectrical impedance technology is quick, affordable, portable and harmless when used non-invasively. All these characteristics make this type of technology very suitable for use in a clinical scenario.

There are some uncertainties about the evolution of the complex bioimpedance during cell swelling and the effect of the frequency on the sensitivity of the resistance and reactance is one of those issues that should be investigated further. The effect of the skull on the non-invasive measurements of the complex bioimpedance, mainly the reactance, should also be addressed.

A clear fact is that, in order to apply the monitoring of changes of complex bioimpedance to detect threatening episodes of hypoxia, multi-frequency measurements have to be performed. But how to perform these measurements, simultaneously or sweeping between certain selected 
frequencies, how many and which frequencies should be monitored, are issues to be approached in order to improve the efficacy of the application of measurement of bioelectrical impedance for the detection of cell swelling.

Independently of these uncertainties, the combination of the monitoring of the electrical bioimpedance of the brain with other monitoring modalities currently in practice, such as EEG activity and cerebral function monitoring (CFM), may improve the effectiveness of current detection methods.

\section{Acknowledgments}

We would like to acknowledge the financial support of the Swedish Research Council (research grant number 2002-5487) and the European Commission (The BIOPATTERN Project, Contract No. 508803).

\section{References}

Ackmann J J, Seitz M A and Geddes L A 1984 Methods of complex impedance measurements in biologic tissue CRC Crit. Rev. Biomed. Eng. 11 281-311

Cole K S 1928 Electric impedance of suspensions of spheres J. Gen. Physiol. 12 29-36

Cole K S 1940 Permeability and impermeability of cell membranes for ions Quant. Biol. 8 110-22

Cole K S 1968 Membranes, Ions, and Impulses. A Chapter of Classical Biophysics (Biophysics Series) (Berkley: University of California Press)

Cole K S 1976 Analogue solution for electrical capacity of membrane-covered cubes in cubic array at high concentration Proc. Natl Acad. Sci. USA 73 4003-6

Cole K S and Cole R H 1941 Dispersion and absorption in dielectrics: I. Alternating-current characteristics J. Chem. Phys. $9341-51$

Cole K S, Li C L and Bak A F 1969 Electrical analogues for tissues Exp. Neurol. 24 459-73

Cotran R S, Kumar V and Robbins S L 1989 Robbins' pathologic basis of disease Cellular Injury and Adaptation 4th edn, ed S L Robbins (Philadelphia, PA: Saunders)

Fricke H 1924 A mathematical treatment of the electrical conductivity of colloids and cell suspensions J. Gen. Physiol. 6 375-83

Fricke H 1925 The electric capacity of suspensions with special reference to blood J. Gen. Physiol. 9 137-52

Geddes L A and Baker L E 1967 The specific resistance of biological material-a compendium of data for the biomedical engineer and physiologist Med. Biol. Eng. 5 271-93

Gunn A J, Gunn T R, Gunning M I, Williams C E and Gluckman P D 1998 Neuroprotection with prolonged head cooling started before postischemic seizures in fetal sheep Pediatrics 102 1098-106

Haueisen J, Tuch D S, Ramon C, Schimpf P H, Wedeen V J, George J S and Belliveau J W 2002 The influence of brain tissue anisotropy on human EEG and MEG Neuroimage 15 159-66

Holder D S 1992 Detection of cerebral ischaemia in the anaesthetised rat by impedance measurement with scalp electrodes: implications for non-invasive imaging of stroke by electrical impedance tomography Clin. Phys. Physiol. Meas. 13 63-75

Jakobsson U 2000 Investigations of the Characteristic Impedance of the Brain and Development of an Impedance Meter for a Wide Frequency Spectrum (Gothenburg: Chalmers University of Technology)

Kanai H, Sakamoto K, Furuya N and Kanai N 2004 Errors on the electrical estimation of intra and extra-cellular fluid volumens XII ICEBI Conference (Gdansk) vol 1 pp 225-8

Klatzo I 1994 Evolution of brain edema concepts Acta Neurochir. Suppl. 60 3-6

Legido A, Katsetos C D, Mishra O P and Delivoria-Papadopoulos M 2000 Perinatal hypoxic ischemic encephalopathy: current and future treatments Int. Pediatr. 15 143-51

Lingwood B E, Dunster K R, Colditz P B and Ward L C 2002 Noninvasive measurement of cerebral bioimpedance for detection of cerebral edema in the neonatal piglet Brain Res. 945 97-105

Lingwood B E, Dunster K R, Healy G N, Ward L C and Colditz P B 2003 Cerebral impedance and neurological outcome following a mild or severe hypoxic/ischemic episode in neonatal piglets Brain Res. 969 160-7

Malmivuo J and Plonsey R 1995 Bioelectromagnetism - Principles and Applications of Bioelectric and Biomagnetic Fields (New York: Oxford University Press) 
Martinsen Ø G, Grimnes S and Schwan H P 2002 Interface phenomena and dielectric properties of biological tissue Encyclopedia of Surface and Colloid Science ed T H Arthur (New York: Dekker)

Maxwell C J 1873 Treatise on Electricity and Magnetism (Oxford: Clarendon)

Penrice J et al 1997 Proton magnetic resonance spectroscopy of the brain during acute hypoxia-ischemia and delayed cerebral energy failure in the newborn piglet Pediatr. Res. 41 795-802

Roelfsema V, Bennet L, George S, Wu D, Guan J, Veerman M and Gunn A J 2004 Window of opportunity of cerebral hypothermia for postischemic white matter injury in the near-term fetal sheep J. Cereb. Blood Flow Metab. 24 877-86

Somjen G G 2001 Mechanisms of spreading depression and hypoxic spreading depression-like depolarization Physiol. Rev. 81 1065-96

Toner I, Taylor K M, Newman S and Smith P L C 1998 Cerebral functional changes following cardiac surgery: neuropsychological and EEG assessment Eur. J. Cardio-Thoracic Surg. 13 13-20

Van Harreveld A 1957 Changes in volume of cortical neuronal elements during asphyxiation Am. J. Physiol. 191 233-42

Vannucci R C and Perlman J M 1997 Interventions for perinatal hypoxic-ischemic encephalopathy Pediatrics 100 1004-14

Williams C E, Gunn A and Gluckman P D 1991 Time course of intracellular edema and epileptiform activity following prenatal cerebral ischemia in sheep Stroke 22 516-21 